\title{
MÉMOIRE FÉMININE DE LA SHOAH EN BELGIQUE : CIEL AVEC TROU NOIR, LE TÉMOIGNAGE DE CAROLINE ALEXANDER
}

\author{
André Bénit \\ Universidad Autónoma de Madrid \\ andre.benit@uam.es
}

\begin{abstract}
RÉSUMÉ : Dans son essai La Shoah de Monsieur Durand (2015), l'écrivaine belge Nathalie Skowronek réfléchit, à partir de son expérience personnelle, à la façon dont la mémoire de la Shoah s'est modifiée au fil du temps et à la question de la transmission future de cet héritage. Une analyse qui se confirme à la lecture $d u$ récit autobiographique, aussi bouleversant que captivant, Ciel avec trou noir (2014) de Caroline Alexander, dans lequel la journaliste culturelle, enfant cachée en Belgique durant la Seconde Guerre mondiale, relate la bienfaisante collaboration entre les deuxième et troisième générations, ainsi que la funeste indifférence, voire l'ignorance crasse de la génération suivante.
\end{abstract}

MOTS CLÉS : Caroline Alexander, Shoah, Auschwitz, Belgique, mémoire, résilience.

\section{MEMORIA FEMENINA DE LA SHOAH EN BÉLGICA: CIEL AVEC TROU NOIR [CIELO CON AGUJERO NEGRO], EL TESTIMONIO DE CAROLINE ALEXANDER}

\footnotetext{
RESUMEN: En su ensayo La Shoah de Monsieur Durand (2015), la escritora belga Nathalie Skowronek reflexiona, a partir de su experiencia personal, sobre cómo la memoria de la Shoah se ha ido modificando a lo largo del tiempo y sobre la transmisión futura de esta herencia. Un análisis que se confirma al leer el relato autobiográfico, tan conmovedor como cautivador, Ciel avec trou noir (2014) de Caroline Alexander, en el que la periodista especializada en temas culturales, niña refugiada y escondida en Bélgica durante la Segunda Guerra mundial, cuenta la necesaria colaboración entre las segunda y tercera generaciones, así como la terrible indiferencia o la peligrosa ignorancia de la generación siguiente.

PALABRAS CLAVE: Caroline Alexander, Shoah, Auschwitz, Bélgica, memoria, resiliencia.
} 


\title{
WOMEN'S MEMORIES OF THE SHOAH IN BELGIUM: CIEL AVEC TROU NOIR [SKY WITH A BLACK HOLE], CAROLINE ALEXANDER'S TESTIMONY
}

\begin{abstract}
In her essay La Shoah de Monsieur Durand (2015) [Monsieur Durand's Shoah], the Belgian writer Nathalie Skowronek reflects, from her personal experience, on the way the memories of the Shoah changed with time and on the question of transmission of this painful legacy. Her analysis is confirmed when one reads Caroline Alexander's Ciel avec trou noir (2014), an autobiographical narrative which is as moving as captivating. Alexander, who is now a cultural journalist, was one of the 'hidden children' during WWII in Belgium. In her narrative, she shows, on the one hand, the positive collaboration between the second and third generations, and on the other hand, the baneful indifference, and even the crass ignorance of the following, 4th, generation.
\end{abstract}

KEYWORDS: Caroline Alexander, Shoah, Auschwitz, Belgium, memories, resilience.

Recibido: 09/02/2020. Aceptado: 28/04/2020

\begin{abstract}
Ceux qui sont morts sont morts, et il leur est bien égal qu'on leur rende hommage. Mais c'est pour nous, les vivants, que cela signifie quelque chose. La mémoire n'est d'aucune utilité à ceux qu'elle honore, mais elle sert à celui qui s'en sert.

Avec elle, je me construis, et avec elle je me console. (Binet 2009: 244)
\end{abstract}

\section{Prologue}

L'impression du trop tard que Nathalie Skowronek (Bruxelles, 1973) ${ }^{1}$ éprouva au cours de la rédaction et lors de la publication de Max, en apparence (2013), un roman dans lequel l'écrivaine belge retraçait le parcours de son grandpère rescapé de la mine de charbon de Jawischowitz - un camp situé à quelques kilomètres d'Auschwitz -, l'amènera à réfléchir, d'une part, à la manière dont la

1. Voir notre étude : « D’Un monde sur mesure au tissage d'un monde à sa mesure. Le défi relevé de Nathalie Skowronek », Intercâmbio, Revue d'Études Françaises / French Studies Journal, $2^{\mathrm{a}}$ série, vol. 12, 2019: 143-193. 
mémoire de la Shoah s'est modifiée au fil du temps, d'autre part, à la question de la transmission future de ce lourd héritage.

Deux ans plus tard, dans son essai intitulé La Shoah de Monsieur Durand (2015), Skowronek montre en effet que, tandis que la première génération, celle des rescapés, préféra se murer dans un profond silence - « désapprendre son histoire pour ne pas en mourir » (14), telle une nécessité existentielle -, que la deuxième, celle de leurs enfants, a grosso modo respecté ce devoir d' " amnésie salutaire » (16) afin de laisser la vie reprendre ses droits, la troisième, la sienne et la dernière à avoir eu accès à la parole vive des survivants, s'est mise à fouiller inlassablement le passé et à « déterre[r] les secrets » (32). Cela, reconnaît-elle, n'empêche nullement certains d'entre eux de « commence[r] à en avoir marre » (36) et surtout de se rendre compte qu'ils agacent la quatrième génération, laquelle souhaite généralement rompre avec cette « contrainte mémorielle » et s'émanciper de ce passé étouffant : « Les descendants sont tiraillés. Devoir de mémoire ou droit à l'oubli ? Une part d'eux est fatiguée d'être fidèle, patiente, coupable. Pourquoi souffrir nous, si eux ont tourné la page ? » (37). Sans doute pourrait-on élargir ce sentiment de lassitude ou de ras-le-bol aux jeunes Allemands, a fortiori s'ils ne sont pas juifs ${ }^{2}$.

Consciente qu'une ère est révolue

\footnotetext{
- Dans le monde qui était le mien, le souvenir et les effets de la Shoah constituaient un système de référence. On s'était construit avec elle ou contre elle. C'était un événement au cœur de nos pensées. Celles des familles juives, bien sûr. Mais pas seulement : l'Europe, elle aussi, s'était construite autour de cet événement (in Audétat 2015) -,
}

que la mémoire de la Shoah ne tétanise plus aujourd'hui comme elle le faisait auparavant, qu'une forme de désintérêt ou de distanciation vis-à-vis de ce passé s'est installée chez les post-millennials, Skowronek s'interroge, non sans quelque inquiétude, sur la façon dont cette génération $\mathrm{Z}$ s'appropriera un tel héritage : certes, la mémoire de la Shoah continuera de se transmettre, mais, dit-elle, « ce ne sera plus la parole incarnée que j'ai entendue dans la bouche de mon grandpère. Elle n'aura donc plus la même charge. Et c'est inévitable. On va tous entrer dans cette histoire nouvelle » (in Audétat 2015). Quelle histoire ? Sans doute est-il trop tôt pour le dire..., selon Skowronek qui se dit néanmoins curieuse de découvrir ce que sera le livre majeur de l'étape suivante, « le livre de l'après-

2. Voir à ce propos, parmi d'autres exemples, l'article de Thomas Wieder "On voit et on entend des choses qu'on n'aurait jamais imaginées avant" : en Allemagne, la mémoire des camps malmenée " (Le Monde, 27 janvier 2020). 
Shoah », écrit par quelqu'un qui n'aura pas « ingurgité la Shoah dès le biberon. Et quand je dis la Shoah, ce sont aussi ses codes, ses interdits, la responsabilité à laquelle elle oblige » (Skowronek 2015) : « On ne saura plus qui parle, ni d'où, mais cela n'aura plus la même importance, ce sera même là la nouveauté : une Shoah pour tous, une Shoah désacralisée, dupliquée, fictionnalisée, mondialisée, analogisée. Une Shoah signée Monsieur Durand » (59).

Cette analyse se confirme pleinement à la lecture du témoignage de Caroline Alexander, Ciel avec trou noir ${ }^{3}$, dans lequel la journaliste, enfant cachée en Belgique durant la Seconde Guerre mondiale, relate l'étroite et bienfaisante collaboration entre les deuxième et troisième générations, ainsi que la funeste indifférence, voire l'ignorance crasse de la génération suivante.

\title{
2. Ciel avec trou noir
}

\subsection{Un sujet non épuisable}

\begin{abstract}
M'assaillent de plus belle mes vieux démons de l'inquiétude, de la jalousie et de la peur de l'abandon. Il y aura toujours, enfoui dans ce qu'il est convenu d'appeler l'inconscient, l'image d'un quai de gare enfumé dans la brume d'une aube incertaine et d'une femme faisant signe, les bras tendus vers une petite fille qui colle son visage sur la vitre d'un train en partance. Ma mère, entrevue pour la dernière fois à l'âge de trois ans. La Seconde Guerre mondiale venait de commencer. Il fallait fuir l'Allemagne où j'étais née juive, donc condamnée... Une femme allemande, 'aryenne', qui allait devenir ma tante par alliance, m'a fait passer en Belgique, zone encore libre. Je n'allais plus jamais revoir cette mère qui disparaîtrait dans les fumées des fours crématoires. Mais elle aura imprimé à jamais, au fin fond de tous les non-dits de ma vie, la peur de l'abandon. (Alexander 2018: 14)
\end{abstract}

Dans ce bref extrait de son roman-autofiction Une vie en miniature (2018) où une journaliste culturelle relate sa vie en souffrance aux côtés d'un mari volage qui la trompe en catimini, du moins jusqu'au jour où elle se découvre la faculté magique de devenir, sur commande, lilliputienne et quasi invisible

- c'est dire, comme l'indique Martine Rouhart, que, s'il s'agit de « l'histoire d'une fuite, fuite de la réalité, d'une vie de couple malheureuse », c'est aussi et avant tout celle d'« une

3. S'agissant d'un récit peu connu, nous nous permettons ici de le présenter d'une manière assez détaillée et d'en citer des fragments parfois un peu longs. 
libération. Une fable métaphorique. Une sorte de leçon sur la nécessité de prendre du recul face à nos problèmes, nos enfermements, nos sujétions, nos dépendances affectives, nos habitudes, notre vie ordinaire. Pour enfin, prendre son destin en main » (Rouhart s.d.) -,

Caroline Alexander (CA) rappelle quelques-uns des épisodes tragiques qui ont profondément marqué son parcours vital, une vie que les lecteurs eurent l'occasion de découvrir quatre ans auparavant dans Ciel avec trou noir (2014), un livre-témoignage qu'elle écrivit En mémoire de Henny-Henriette, ma mère et de Gert, mon frère / ’̀ Meta, ma petite maman (10), et qu'elle dédicace À Morgane, ma fille, dont la tendre vigilance me fut si précieuse (232) .

Dans ce récit qui se lit comme un roman à suspense et qu'avec Lydia Flem, on pourrait qualifier de «non-fiction novel, un roman qui ne serait pas une fiction, une vérité qui serait de la littérature » (Flem 2009: 103), la narratrice relate par petites touches, dans une chronologie bousculée, comme au gré d'une mémoire s'efforçant d'ordonner patiemment les souvenirs, fragment par fragment - le plus ancien étant daté du 3 janvier 1946, le plus récent du 5 avril 2011 -, la longue enquête et les nombreuses démarches qu'elle effectua tous azimuts, avec ténacité, pendant soixante-cinq ans, à la recherche de la vérité sur son passé et celui d'une partie de sa famille disparue dans le tourbillon de l'Holocauste.

Comme l'indique en quatrième de couverture le journaliste Jacques Bredael,

De Paris à Mönchengladbach, de Leicester à Bruxelles, se reconstitue peu à peu l'histoire d'une petite fille née dans un bordel, sauvée par une 'maman Meta' d'adoption, et mise à l'abri alors que sa vraie maman disparaît dans le brouillard de la déportation. Où est-elle morte ? Et comment ? C'est la réponse indispensable à ces questions - car le deuil est à ce prix - qui se dessine au fil d'une recherche menée pendant plus d'un demi-siècle.

Rétorquant l'argument de ceux qui, « avec un insidieux manque de bienveillance », s'exclameraient " encore un livre sur ce sujet ! », Pierre Mertens, dans une préface intitulée « Le goût de (sur)vivre », souligne que le sujet d'un livre comme celui-ci n'est guère épuisé et ne saurait l'être : « il n'est, à vrai dire, pas épuisable » (5) ; car, dit-il, « on ne viendra jamais à bout de l'exploration du continent de la mort industrielle et du mal absolu. Il y aura,

4. Édité lui aussi à Bruxelles aux Éditions MEO (Monde-Édition-Ouverture), préfacé par Pierre Mertens et couronné par le prix Emma Martin décerné par l'Association des Écrivains belges de Langue française. 
hélas encore $[\ldots]$ des multitudes de façons d'aborder le thème où le vingtième siècle s'est englouti » (6).

Dans cette étude, évitant de reprendre les notions maintes fois analysées dans de nombreux travaux, nous nous proposons d'agencer les multiples pièces éparses et enchevêtrées de ce " puzzle autobiographique en forme d'énigme " (Lafon), de ce récit haletant et captivant - on aimerait aussi l'imaginer interpellant en cette période de resurgissement des partis fascistes un peu partout en Europe ! -, et ce afin de retracer l'itinéraire bouleversant de cette femme qui, après avoir miraculeusement échappé au destin réservé à quantité de membres de la communauté à laquelle elle appartenait, sut mener à bien la quête de l'indispensable vérité, essentielle pour pouvoir faire son deuil. Comme nous le verrons, une telle relation lui a permis de méditer sur des sujets récurrents dans l'abondante littérature de/sur l'Holocauste, que celle-ci soit autobiographique, autofictionnelle, ou pure fiction : le silence et l'impuissance des rescapés à témoigner leur vécu ${ }^{5}$, la difficile transmission générationnelle et le sentiment de culpabilité intergénérationnelle, l'effacement de la mémoire - « la mémoire menacée » (Todorov 2004: 9) - et les dangers de l'oubli, la lâcheté des uns vs la solidarité des autres, le pardon et la réconciliation, les traumatismes divers des descendants, l'exil en terre francophone, la résilience par l'écriture...

\subsection{Le pèlerinage de Mönchengladbach en 1989}

En août 1989, Caroline Alexander participe à « un singulier pèlerinage » (2014: 13) organisé par la mairie de Mönchengladbach, la ville où elle est née le 25 février 1936 et qu'elle quitta clandestinement fin 1939 à destination de la Belgique où elle fut « enfant cachée » pendant la guerre : «1936, l'année du Front Populaire, de Léon Blum et des premiers congés payés, une année de chants d'espoir pour la France. Mais pas pour moi. Moi, je suis née dans un endroit où le mot espoir ${ }^{6}$ était alors interdit de séjour pour les gens de mon espèce » (14-15).

Suite à la publication de l'ouvrage Juden in Mönchengladbach (1988) dans lequel le professeur Gustave Erckens retraçait l'odyssée du judaïsme dans cette

5. À cet égard, ce qui concerne la Belgique, voir notre contribution « Au-delà des multiples silences... Fictionnaliser la Shoah pour en surmonter la douleur et en perpétuer le souvenir. Le défi de Vincent Engel et de Françoise Lalande-Keil », dans le dossier monographique Le silence dans l'écriture de la Shoah, Çédille, Revista de estudios franceses, otoño 2015: 65-96.

6. C'est nous qui soulignons. 
région de Rhénanie-du-Nord-Westphalie depuis le Moyen-Âge jusqu'à la Shoah, la Gesellschaft für christlich-jüdische Zusammenarbeit (Société pour la coopération judéo-chrétienne) a en effet proposé aux autorités municipales de partir à la recherche des survivants locaux du génocide, essaimés de par le monde, et de les convier à un retour au pays afin d'honorer la mémoire de ces hommes, femmes et enfants qui, en raison de leur appartenance à la communauté juive, furent, dès les diktats raciaux émis en 1933 par le national-socialisme triomphant, différenciés des autres citoyens, avant d'être "parqués, ghettoïsés, déportés, gazés, brûlés, assassinés » (13). Ce sont cent soixante-quatre " âmes de la diaspora » (47), tous « parents et cousins des fantômes des nuits et des brouillards » (49), qui répondirent " présentes » à cette invitation de la municipalité « à mêler [leurs] nouvelles racines aux anciennes » (18).

C'est lors de cette rencontre - à laquelle elle est reçue non à titre personnel mais en tant qu'accompagnatrice de sa tante Meta Leven, la jeune sœur de sa mère, devenue sa " petite maman d'adoption »-que cette journaliste parisienne de 53 ans apprend enfin, outre le lieu, l'heure exacte de sa naissance : trois heures du matin. Un élément apparemment anodin mais qui ne l'est nullement : d'une part, il permet à l'un de ses amis mordu d'astrologie de lui indiquer son ascendant : "Sagittaire, un signe de feu. Poissons-Sagittaire, l'eau et le feu... Il trouve que ça me ressemble » (14) ; d'autre part, à l'intéressée ellemême d'évoquer un souvenir certes vieux de vingt-cinq ans mais demeuré profondément gravé dans sa mémoire : la révélation, par « un étrange bonhomme piqué d'ésotérisme », de la présence insolite dans sa carte céleste de " quelque chose comme un manque ou une disparition » (14). Ainsi, tout au long de son récit, CA relate par bribes la conversation troublante qu'elle eut, un soir de juin 1964, avec le singulier gérant antisémite et fasciste de la librairie du théâtre de l'Ambigu à Paris. Ce dernier, bien qu'elle n'ait pu lui fournir cette donnée capitale - l'heure de sa naissance -, avait insisté pour « faire son thème astral », une étude au terme de laquelle il lui dévoila l'existence d'un creux qui, affirmait-il, très tôt dans sa vie avait tout freiné et embrouillé : un " trou noir qui vous aurait avalée dès la petite enfance. Un ciel avec une éclipse... Un foyer sacrifié... Père absent... Mère... » (103), « un manque, ce qu'on appelle un sacrificiel foyer, pour ainsi dire une famille sacrifiée, puis, entre trois et cinq ans, une éclipse totale qui vous a plongée dans la nuit... Il y a un vide dans votre ciel, mademoiselle, un trou noir... Je ne vois pas... » (113). À l'écoute de ces paroles obscures, même si elle s'était sentie en sécurité auprès de l'homme goy qu'elle venait d'épouser, CA n'avait pu s'empêcher d'être tout à coup étreinte par la peur : 
Peur du passé inconnu, peur du bruit de bottes, des synagogues en flammes, des livres brûlés, des fumées, des fours crématoires, peur d'être condamnée à être abandonnée, hier, aujourd'hui, demain. [...]. Qu'il cherche donc mon foyer éclipsé, mon manque, mon trou noir, pauvre libraire nostalgique des nazis! Qu'il les cherche dans Wagner et les chants de gloire du IIIe Reich. Il ne les trouvera pas. Moi, je sais où ils sont. (114)

Plus tard, elle lui avouera cependant qu'il avait vu juste : « - Le foyer sacrifié, c'est ma mère. [...]. Elle est morte à Auschwitz »; en réalité, ajoute-telle pour le lecteur de son récit mémoriel, à cette époque elle ignorait tout de la date et du lieu où sa mère Henny-Henriette et son frère Gert avaient disparu ensemble ou séparés ? : « Je dis Auschwitz, car dans ce nom symbole résonnent les noms de tous les autres camps $»(142)^{7}$.

« Quand ? Où ? Comment? » sa mère et son frère aîné sont-ils morts (13). Cette triple et douloureuse énigme qu'elle ne cesse de se poser et de ressasser depuis qu'elle a dix ans, toutes les questions qui obsèdent ses pensées depuis qu'en 1946 elle comprit qu'elle ne reverrait plus jamais ces êtres dont elle n'a aucun souvenir, il ne lui faudra pas moins de soixante-cinq années pour en trouver les réponses définitives, des versions contradictoires lui ayant été livrées par les instances et les personnes auxquelles elle s'était adressée durant ces dernières décennies.

De fait, lors du séjour à Mönchengladbach en 1989, après avoir appris l'heure et le lieu de sa naissance : "Trois heures du matin. 10A, Gasthausstrasse ! (55), « la rue des putes » $(90) !^{8}$ - ce qui lève une partie du mystère entourant « ce qui fut le début de [s]on existence » (19) -, à la vue du malaise dont est soudainement victime l'adjoint au maire qui tient en mains un certificat la concernant, CA sent aussitôt quelque chose se déchirer en elle : " une très, très vieille blessure que je croyais cicatrisée fait encore mal $»(81)$. Au cours de la visite ultérieure de l'exposition organisée dans les locaux du «Haus Zoar », la maison paroissiale de l'Eglise évangélique, elle découvre en effet, sur une liste, la présence d'un

7. Pour beaucoup de descendants, Auschwitz est en effet devenu une sorte de formule générique englobant l'ensemble du génocide : « Un terme métonymique », pour reprendre l'analyse de l'historienne française Annette Wieviorka dans son essai L'heure d'exactitude: Histoire, mémoire, témoignage (2011).

8. «Je suis née en 1936 à Mönchengladbach, à trois heures du matin, entre le vieux marché et l'abbaye. Pas dans une clinique. Les maternités de la région étaient interdites aux juives. Je suis née au premier étage du 10A, de la rue des Auberges - Gasthausstrasse -, la rue qui accueille, la rue des putes et des maisons closes. [...] en 1936 à Mönchengladbach, les juifs n'avaient plus le droit d'habiter les beaux quartiers et leurs femmes ne pouvaient accoucher dans les hôpitaux, si bien que, cette année-là, les petites filles juives naissaient près des bordels » (Alexander 2014: 15). 
troisième nom aux côtés de ceux de sa mère et de son frère, tous deux déportés au ghetto de Riga en décembre 1941 : le sien, avec la mention : « Née le 25 février 1936, déportée en 1941 à l'âge de cinq ans » (89). Tout cela, on peut l'imaginer, au grand désespoir et à l'effarement de la jeune archiviste qui, après s'être donné tant de mal pour collecter tous ces renseignements, a fait « l'erreur qu'il ne fallait surtout pas commettre, enterrer un vivant parmi les morts assassinés » (89). Car la même bourde figure sur les bristols, sur le catalogue de l'exposition, sur «Le Livre du Souvenir » et même dans le premier volume de l'ouvrage du Dr Erckens : « La vie est devenue furieusement cocasse à Mönchengladbach, en ce mois d'août $1989 »(90)$, ironise a posteriori la narratrice.

Parmi les retrouvailles les plus émouvantes, celle de son cousin germain Manfred, matricule A1663, « miraculeusement revenu des camps » (24), et de sa femme Christel « qui ne connaît de son martyre que les bribes de phrases et les cris lâchés chaque nuit de cauchemar depuis quarante ans » (146-147). Arrêté le 10 novembre 1938, le jour de l'incendie de la synagogue de la ville, et déporté à l'âge de huit ans avec un millier d'enfants - il est le seul de ceux-ci à " avoir survécu à l'enfer des camps » (148) -, dès sa libération Manfred est revenu dans sa région d'origine afin d'y entretenir les tombes en ruine ; aussi, c'est à lui, « le gardien des cimetières » (48), que les visiteurs doivent s'adresser pour obtenir la clé des deux cimetières juifs où ils iront se recueillir. Lui qui jusqu'à cette date a toujours refusé de parler de son séjour dans les camps - Terezin, Auschwitz, Birkenau et Buchenwald -, accepte enfin, à l'invitation du pasteur, de raconter aux enfants présents, des adolescents en compagnie de leur professeur, quelquesunes des choses qu'il y a vécues, non sans toutefois leur dire en préambule : "On en parle jamais, nous, ceux qui en sont revenus, c'est pas la peine, y en qui nous croiraient même pas, parce que c'est tellement pas croyable qu'on peut pas l'expliquer. Personne peut l'expliquer. J'ai jamais raconté, pas même à ma femme, ni à ma fille $[\ldots] »(151)$.

Dans un des chapitres les plus poignants de l'ouvrage de Caroline Alexander, Manfred relate donc avec sobriété et retenue la rencontre avec sa grand-mère maternelle, Oma Adèle, à Terezin ; le calvaire de la déportation à Auschwitz les séances de sélection sous la houlette du docteur Mengele et les chambres à gaz, les mauvais traitements et les travaux forcés, les exécutions sommaires et les suicides de ceux qui préféraient se jeter sur les barbelés électrifiés - ; les transferts d'un camp à l'autre, le froid glacial, puis la libération du camp de Buchenwald, son arrivée à Paris où, en raison de sa nationalité allemande, il fut insulté et arrêté... « Je suis le seul à être revenu. Voilà, vous en savez un peu 
maintenant, ne l'oubliez pas ", conclut-il (160), comme en écho au discours « toujours sage, toujours passionné pourtant» du rabbin venu de Buenos Aires - il a obtenu son diplôme de rabbinat à Berlin en mars 1939 -, en compagnie de sa fille Ruth :

- Nous sommes venus. Notre raison nous disait 'n'y va pas', notre cœur murmurait 'vas-y'. Ceux qui sont ici ont écouté leur cœur. Ils ont eu raison, car, dans leurs bagages, ils ont apporté leur mémoire. Cette mémoire sans laquelle la réconciliation implorée par nos hôtes est impossible. Pardonnons, mais n'oublions pas, forgive, not forget. (127-128)

C'est également au cours de ce « voyage-pèlerinage de 1989 » (45) que CA écoutera le témoignage de Hilde Sherman, une survivante du ghetto de Riga, selon qui Henny-Henriette et Gert, qu'elle y avait côtoyés, auraient réussi à survivre à la misère, à la faim, aux traitements ignobles, jusqu'au mois d'août 45 et auraient été abattus dans la forêt de Hochwald (179), aux portes du camp, et jetés dans une fosse improvisée, à la veille de l'arrivée des troupes russes, par les officiers SS en déroute mais désireux de faire quelques ravages supplémentaires. Bien que cette rescapée lui assurât que, durant leur détention, sa mère et son frère n'avaient pas été trop malheureux (78), « ce fut un choc » (45), car, signale $\mathrm{CA}$, cette révélation dont elle mit longtemps à accepter la réalité « avait peuplé [s]es nuits de cauchemars »(105) ; elle lui avait en effet subitement enlevé "l'illusion de [leur] fin rapide, et pour ainsi dire, consolante » qu'elle s'était imaginée - « allez savoir pourquoi »- lorsque la nouvelle de leur mort lui avait été confirmée après la guerre : en 1946-47, la fillette de dix ans avait fantasmé qu'à son arrivée au camp de Riga, les nazis avaient imposé à sa mère le « choix de Sophie » :

se séparer de son enfant ou périr avec lui. Et tout naturellement je pensais qu'ils avaient été aussitôt anéantis. Cette thèse que je m'étais fabriquée comme la pièce d'un puzzle mouvant m'épargnait le questionnement de la vie, des souffrances, des humiliations des camps de concentration. (45)

Quarante ans plus tard, la rescapée de Riga lui avait donc ravi « cette maigre consolation » :

Ce fut une secousse dont je me remis difficilement. Tout à coup le rêve que j'avais fait à la Libération de voir ma mère débarquer et sonner à notre porte prenait un sens nouveau. Je 
réalisais que cela aurait donc pu, que c'eût été de l'ordre du possible, si ce SS en déroute ne s'était pas offert une ultime bestialité. (105-106)

Lors de ce premier retour à Mönchengladbach, CA se souviendra à plusieurs reprises, d'une part, du moment de la douloureuse séparation, un matin de novembre 1939 où tombait une pluie glacée sur le quai de la gare, d'avec sa mère en pleurs - « [elle] a trente-sept ans, elle n'aura jamais de rides » - et son grand frère de sept ans - «[il] n'aura jamais de cheveux blancs » (111) -, alors qu'elle-même dont « [le] corps se vide de ses larmes » (174) n'a que trois ans et sept mois : « ils m'ont laissé(e) partir sous un gros bonnet de laine et eux, s'en sont allés mourir sans moi » (58); d'autre part, de sa fuite en Belgique en compagnie de « Martha Stäuber, la blonde aryenne qui sauvera ma vie au risque de la sienne » (111) et qui, après la guerre, deviendra sa tante : elle épousera Otto, son oncle survivant des camps d'AuschwitzBirkenau et de Buchenwald où il fut déporté sur dénonciation de leur logeuse, collabo de la ville d'Anvers, où ils avaient trouvé refuge après avoir pu quitter l'Allemagne in extremis (83).

\subsection{Les années belges: les années de formation}

À son arrivée en Belgique, CA est recueillie, en principe provisoirement, par sa tante Meta, en attendant que la famille se réunisse - peut-être - en Angleterre où son père, Alfred Alexander, a fui en 1938, sans femme ni enfants.

Durant l'Occupation, la fillette connaîtra plusieurs cachettes : à Anvers, dans l'appartement situé dans le ghetto juif ; à Bruxelles, près de la place Liedts, puis dans la rue des Sicambres à Etterbeek ${ }^{9}$; près de Bruges, dans un pensionnat où « les bonnes sœurs n'arrêtaient pas de dire que les méchants juifs avaient tué le gentil petit Jésus » (75) ; avant que le père Bruno Reynders ${ }^{10}$ ne lui trouve d'autres refuges, à la campagne, notamment chez des fermiers dans le village Sorle-Saint-Gerry (sic pour Solre-Saint-Géry, en Hainaut) où elle vécut les premières heures de la Libération (61).

9. Bruxelles et Anvers sont les villes où, en Belgique, se concentre historiquement la majorité des immigrés juifs.

10. « [...] un prêtre en civil, entré dans la Résistance. Un moine bénédictin, le père Bruno Reynders. Il avait provisoirement accroché sa soutane au vestiaire de l'abbaye d'Ottignies pour, déguisé en civil, sauver 390 enfants juifs. Il porta de multiples identités, toutes fausses. La dernière, la vraie, lui fut donnée après sa mort : 'Héros de la Résistance, et Juste des Nations' » (52). 
Quand l'espoir de voir revenir sa mère et son frère fut officiellement anéanti, sa tante Meta - « ma petite maman comme je l'appelais déjà » (59) - décida de l'adopter, en accord avec Julius, « son mari rencontré durant l'occupation et qui avait remplacé mon père sans jamais rien demander en échange » (41-42). Ce père géniteur qui, dira-t-elle plus tard, prit lâchement la fuite en 1938 et dont elle ne découvrira l'existence qu'à l'âge de douze ans, elle ne lui pardonnera jamais d'avoir abandonné sa famille «à l'hydre nazie » (38). Rien de surprenant donc à ce qu'elle ait ressenti un profond soulagement lorsque, le 1er avril 1949, leur rendant visite à Bruxelles - « Nous habitions au numéro 100 de l'avenue Winston Churchill » (64) -, il leur annonça d'une part qu'il valait mieux que sa fille poursuive ses études en Belgique, d'autre part qu'elle recevrait prochainement un passeport anglais ${ }^{11}$ grâce auquel elle pourrait aller passer ses vacances auprès de lui, outre-Manche, afin d'y apprendre la langue de Shakespeare : « Je l'aurais presque embrassé pour tout cela, ce père dont j'avais honte ! », signale CA tout heureuse alors de « sortir de [s]on no man's land identitaire des apatrides $»(65-66)^{12}$.

C'est ainsi que, durant l'été 1950, l'adolescente se rend à Leicester. La veille de son départ, elle rêve qu'elle y est accueillie par un père aimant :

Toujours ce besoin d'être aimée et rassurée de l'amour qu'on me porte. De quand datent ce doute permanent, cette nécessité d'être rassurée ? De la vision d'une femme sur le quai d'une gare un matin d'automne en 1939, l'unique image-souvenir que j'ai de ma mère ? Pourquoi notre mémoire d'enfance est-elle si fugitive ? (96)

Les retrouvailles tourneront cependant vite au cauchemar, Caroline ne tardant guère à comprendre qu'elle n'est ni bienvenue ni désirée :

Un jour où j'étais assise à côté d'Alfred, je vis [dans la théière] mon image se poser à côté de la sienne. La ressemblance était flagrante, le front, l'arc des sourcils, le regard sombre.

11. Entretemps, son père, né à St. Wendel, dans la Saar, le 12 septembre 1905, est devenu citoyen britannique « pour avoir servi dans l'armée de Sa Majesté » (63) ; «Ça aussi je le savais et le portais depuis tant d'années comme une tare secrète » (112).

12. «Déchue de la nationalité allemande pour cause de judéité pratiquement à ma naissance, clandestine pendant la guerre, orpheline après, avec un prénom qui sonnait 'boche' - essayez donc de prononcer correctement Ingeborg en français, c'est un vrai jeu de massacre. Heureusement il y avait Caroline après (à l'origine avec un $\mathrm{K}$ que je camouflai), mais Caroline, ce n'était pas à la mode. Les filles de ma classe se prénommaient Jacqueline, Nicole, Élisabeth, Nadine, Anne ou Marie. J'étais une petite juive survivante de l'Holocauste, mais mon nom et mon prénom étaient de consonance allemande et m'apparentaient à la nation barbare qui avait mis le monde à feu et à sang » (65). 
Un sentiment d'humiliation me fit reculer et changer de place. Je ne voulais pas ressembler à cet homme-là. Je voulais qu'on retrouve sur moi les traits de ma mère disparue. J'évitai depuis ce jour de me trouver côte à côte avec lui. (100)

Trois ans et demi plus tard, tout à la fin du mois de décembre 1953, Meta reçoit une lettre d'Alfred leur annonçant son prochain départ pour l'Argentine. Le 2 janvier 1954, la jeune fille de dix-huit ans retraverse la Manche, à contrecœur, pour s'en aller lui faire ses adieux. Sur place, les voisins l'informent que son père est hospitalisé suite à une crise cardiaque. Le lendemain matin, en apprenant son décès dans la nuit, elle se sent traversée, dit-elle, par « des pensées confuses » : « Mon père est mort! Je n'aurai plus jamais à craindre qu'il m'arrache à Meta, au nid que je me suis fabriqué dans son logis » (167).

Le sentiment qu'elle éprouve à ce moment diffère radicalement de celui qui l'envahit six mois plus tôt lors du décès, le 2 juillet 1953, à 84 ans, de sa grandmère maternelle, Oma Adèle, avec laquelle elle avait partagé d'intenses moments de connivence depuis que Marta et Julius étaient allés la chercher en Suisse où la Croix-Rouge internationale l'avait ramenée de Terezin-Theresienstadt, « ce camp en trompe-l'œil près de Prague où les nazis ont essayé de donner une image de marque 'humanitaire' à leur industrie [de la mort] » (222). Lors de leurs conversations, se souvient $\mathrm{CA}$, cette grand-mère douce et discrète, qui ne parlait que l'allemand,

ne se plaignait jamais de rien et ne disait rien, ou à peu près rien, de ses années de camp de concentration. Jamais elle ne fit allusion à tous ces musiciens, Viktor Ullman et les autres, qui y avaient été internés, ni de ce statut bidon de camp réservé aux artistes... La seule chose qu'elle m'ait confiée c'est le fait que là-bas elle mangeait les épluchures de pommes de terre. Sinon rien, comme tous ceux qui sont revenus de ces enfers. (60)

Elle préférait en effet l'entretenir de ses jeunes années, de son village d'Odenkirchen, de son mari Max, décédé en 1930, et également de la musique qu'elle avait découverte lors de l'année passée à Aix-la-Chapelle, au service d'un avocat, un ami du compositeur et diplomate Ignacy Paderewski, en vue d'y apprendre les tâches ménagères (60 et 120). De sa fille aînée Henny-Henriette, Oma ne disait presque rien, " sinon qu'elle était foncièrement bonne - bonne poire même - et qu'elle avait de belles jambes qu'on reconnaissait de loin. Maigre biographie. Mais encore? » (62), s'interroge CA, d'autant que sa tante Meta ne lui en disait pas davantage malgré les questions qu'elle lui posait : « elle 
n'aimait pas parler du passé, elle n'aimait pas se souvenir, seul comptait pour elle le présent et après. Quand une page était tournée, quel besoin pour elle de feuilleter le livre à l'envers ? » $(60)^{13}$.

À l'époque, CA est sur le point de terminer ses études secondaires à l'Athénée Royale d'Uccle, le premier lycée mixte de la capitale belge, où, se rappelle-telle, la trouvant sympathique, le professeur de français de sixième l'appelait " Gigi » en référence à Colette dont elle dévorait les romans. "Bonne élève et délurée » (66), adorant la langue française dont elle avait décidé de faire « [s]a patrie spirituelle » (167), elle avait donc pour cet enseignant un petit air de Gigi, un surnom, dit-elle, que ses anciens camarades de la troupe de théâtre rattachée à l'Université Libre de Bruxelles où elle fit des études de droit - dont Raymond Passauro $^{14}$ qui deviendra son compagnon bien des années après, en 2004 prennent encore un plaisir complice à utiliser pour la nommer !

\subsection{La résilience par l'écriture}

Installée quelques années plus tard à Paris où elle commença par exercer différentes activités dans le monde du cinéma et du théâtre, c'est à partir de 1965, dans le journalisme et l'écriture, que CA trouvera bientôt sa voie et le chemin de la résilience. Dans Un merveilleux malheur, le neuropsychiatre Boris Cyrulnik, né en 1937 dans une famille d'immigrés juifs d'Europe orientale et dont la destinée est en quelques points comparable à celle de Caroline Alexander - ses parents furent déportés de Drancy à Auschwitz et lui, orphelin, fut recueilli à Paris par une tante maternelle - définit de la sorte ce concept qu'il fut l'un des premiers à développer en France : « la capacité à réussir à vivre et à se développer positivement de manière acceptable en dépit du stress ou d'une adversité qui comporte normalement un risque grave d'une issue négative » (Cyrulnik 1999: 10). Dans un essai récent, La nuit, j'écrirai des soleils (2019), Cyrulnik, qui confie par ailleurs que c'est l'expérience personnelle traumatisante qui l'a stimulé à devenir psychiatre, analyse, à l'aune de ses propres souffrances, les déchirures de nombreux grands écrivains, principalement des XIXe et XXe siècles (parmi lesquels les classiques Lévi, Perec, Gary, Veil, pour le sujet qui nous occupe ici), et ce pour mieux convaincre tout un chacun des énormes bienfaits et des vertus

13. Meta mourra en 1996 d'un cancer qui l'emportera en trois mois, douze ans après son mari Julius. Elle est enterrée au cimetière juif de Kraainem (en région bruxelloise).

14. C'est lui qui a créé les illustrations - des variations originales sur le thème de l'étoile de David - qui ponctuent chacun des chapitres du texte de Caroline Alexander (http://passauro.com/). 
reconstructives de l'écriture. C'est dire, pour en revenir au récit de CA, que le libraire-trappiste-fasciste de l'Ambigu qui, l'année précédente, lui avait prédit que sa vie professionnelle était sur le point de virer et qu'elle y brillerait un jour, ne s'était guère fourvoyé : « De comédienne je devins journaliste, l'écriture devint ma parole et le resta » (177).

Début décembre 2005, CA reçoit un appel téléphonique d'une certaine Doris Schilly, une historienne spécialisée dans la vie des cités et qui, un an plus tôt, a publié un livre sur la ville de Mönchengladbach où elle est née en 1959 : Mönchengladbacher Zeitgeschichte(n): 1914 bis 1983 (L'histoire contemporaine de Mönchengladbach : de 1914 à 1983) (2004). Au cours de son travail réalisé à partir d'interviews, Schilly a constaté qu'avant le nazisme, la ville comptait de très nombreux citoyens juifs, des gens de tous les milieux (ouvriers, professions libérales, artistes, commerçants, penseurs, industriels) « qui tissaient une citoyenneté à la fois à part et parfaitement intégrée » $(35)^{15}$; aussi, dans sa recherche d'informations, s'est-elle mise en quête de témoins survivants afin de reconstituer ce qu'avaient pu être leurs vies avant 1945. C'est alors qu'elle apprit l'existence du pèlerinage de 1989 et que lui furent remis des documents et adresses, dont celle de CA. Bien entendu, cette dernière lui confiera n'avoir nulle souvenance de cette époque mais garder dans son débarras un carton rempli de photos et de souvenirs, « trop lourd de secrets », auquel, dit-elle, elle n'aime guère toucher (35-36).

Rendez-vous est pris à Paris. Schilly y restera trois jours : « trois jours d'interrogations qui devenaient ici ou là des interrogatoires ", « trois jours que je n'avais pas imaginés si difficiles à vivre et qui se transformèrent parfois en descente infernale. En spirale, en glissades avec de soudains trous d'air » (36), confie $\mathrm{CA}$. Au cours de leurs entrevues, elle dévoilera en effet à cette femme qui, il y a peu, lui était inconnue « des choses jusqu'alors tues » (26), des «pages jamais écrites » de son enfance et de son adolescence (59), des pans de vie qu'elle a toujours portés en elle " comme une tare inavouable », " le non-dit de soixante années de honte ", entre autres le comportement de l'homme qui lui légua son patronyme et dont elle ne pardonnera jamais la pleutrerie (37). Toutefois, dit-elle, « Doris Schilly, sans le chercher, sans le vouloir et sans le savoir, a fait tomber une cloison emmurée de ma mémoire » (165).

À la mi-février 2006, CA obtient un premier décryptage de leurs entretiens - « À le lire et relire, mes vieilles angoisses grattent à nouveau à la porte "

15. Le pourcentage de Juifs parmi les commerçants de haut niveau en 1933 à Mönchengladbach était de plus de $65 \%$ (39). 
(83) -, puis un second : « Nouvelles corrections, précisions, nouveaux voiles de pudeur », indique CA qui se demande pourquoi elle lui a parlé de son père, une question à laquelle Doris répondra ainsi : «On ne peut pas toujours se mentir à soi-même ». Assurément l'argument fait mouche : « Je laisserai les passages le concernant. Je laisserai dire sa mort, ce soulagement » (83), écrit CA, qui relit également les confidences qu'elle lui fit à propos de la vaine attente de sa mère, des images et des sensations retenues lors de son sauvetage in extremis en novembre $1939 \ldots$

Dans l'ouvrage qu'elle tirera fin 2006 des témoignages recueillis : Mitten unter uns - jüdisches Leben in Mönchengladbach (Parmi nous - la vie des Juifs de Mönchengladbach), l'historienne allemande retranscrira fidèlement le récit, fait par CA, de la disparition de sa mère et de son frère, tel qu'il lui fut conté en 1989 par Hilde Sherman : "C'est en 1947 ou 48 que nous apprîmes qu'il n'y avait plus aucun espoir de retrouver ma mère et mon frère, qu'ils étaient morts tous les deux à Riga. Ce n'est que quarante ans plus tard, lors de cette rencontre organisée à Mönchengladbach que je sus quand et comment ils avaient été éliminés » (84).

\subsection{Les "Stolpersteine"}

Le 10 septembre 2007, le préposé aux archives de la mairie de Mönchengladbach contacte CA en vue de l'organisation de la cérémonie qui aura lieu deux mois plus tard : la pose de pierres commémoratives à la mémoire des Juifs de la ville déportés et assassinés, des Stolpersteine créées par le peintre et sculpteur Günter Demning, « dit-on, pour oublier et s'amender du passé politique de son père » (44), des « pavés à trébucher » destinés à être scellés sur le trottoir devant le dernier domicile connu des victimes et qui « obligeront les passants qui les verront ou les heurteront à s'incliner pour pouvoir les déchiffrer» (24). Audit préposé apparemment perplexe, CA livre les informations dont elle dispose, ce qu'elle apprit quelque vingt ans auparavant de Hilde Sherman et « qui depuis, dit-elle, n'a cessé de [la] hanter » (53).

Un mois plus tard, dans une lettre datée du 8 octobre 2007, Doris Schilly la prie de bien vouloir vérifier le texte qui sera gravé sur les deux pierres. À sa grande surprise, il y est écrit que sa mère et son frère sont morts à Auschwitz! " D'où diable vient cette nouvelle version? » (78). CA apprendra par sa correspondante que cette version du préposé aux archives de la ville de Mönchengladbach se base sur le témoignage d'un certain monsieur Winter, un rescapé vivant à New 
York et qui, dans un courrier adressé à ladite municipalité, affirme que le camp de Riga fut dissous en 1943, que les prisonniers survivants furent acheminés vers d'autres camps et qu'Henriette Alexander, son fils et sa fille furent convoyés de Riga à Auschwitz... !

Je me cache, je me terre dans ma peur. Peur de savoir, de refaire ce chemin encore une fois, d'avoir à inventer d'autres paysages, d'imaginer des wagons à bestiaux où ma mère et mon frère seraient entassés pour être acheminés vers ce portail symbole qui aujourd'hui figure sur tant de plaques, de textes, de films. Avec ces mots surplombant l'allée centrale 'Arbeit macht frei'. / Je ne veux pas. Je ne peux pas. Je m'enferme. Pourrais-je un jour faire le deuil de tout cela? (79)

Comme prévu, le 12 novembre 2007, CA et Raymond Passauro sont accueillis à Mönchengladbach par Doris Schilly et son père, lequel, lors de la parution du livre de sa fille un an plus tôt, s'était proposé de parrainer les "Stolpersteine" de HennyHenriette et de Gert. Le lendemain matin, à la cérémonie de la pose des pierres, « symboles des deux manques qui ont marqué ma vie » (26) et par lesquelles sa mère et son frère " emportés dans la tourmente seraient ici immortalisés », CA arrive quelque peu en avance, « avec un sentiment d'absence » (24), se demandant si elle a raison d'être là : " Je me sens prise au piège de cette initiative lancée il y a un an » (26). Heureusement, sur les lieux, elle retrouve la fille et la petite-fille de son cousin Manfred ainsi que deux femmes qui étaient présentes lors de « l'étrange pèlerinage » (24) de 1989 ; mais elle y est « surtout » accompagnée de sa fille arrivée de Paris : «Elle a voulu venir. [...] Elle dit que c'est important pour elle. Elle veut savoir. Tout savoir » (27), précise CA, avant d'ajouter que les entretiens téléphoniques qu'elle a eus dernièrement avec le chargé des états civils de la mairie l'ont convaincue qu'il existe « une autre vérité à débusquer » (27).

De fait, c'est sur une douloureuse interrogation que, quelques semaines auparavant, s'est rouverte « une plaie » qu'elle pensait pourtant avoir réussi à panser après le voyage-pèlerinage de 1989 au cours duquel lui avaient été racontées les circonstances du décès de ses proches. Aussi, à la différence des autres plaques de cuivre posées par-ci par-là par Günter Demning, celles qui seront placées devant l'ex-10A de la Gasthausstrasse ne mentionnent ni le lieu ni la date de leur mort qui « restent à découvrir » : « Hier Wohnte HENRIETTE ALEXANDER geb. Leven JG. 1902 Deportiert 1941 RIGA ermordet (45) ${ }^{16}$.

16. « Ici habita Henriette Alexander, née Leven en 1902, déportée en 1941 à Riga, assassinée. » 
Mes doigts glissent sur l'espace resté vide... Un manque encore. Une question qui brusquement m'obsède. Où sont-ils morts ? (70)

Sur les deux plaques de cuivre, les 'Stolpersteine' du sculpteur Günter Demning, sont gravés les noms et dates de naissance de ma mère et de mon frère, suivis des indications : 1941, déportés à Riga. Et, en dessous, 'ermordet'. Ni date, ni lieu. Je n'ai pas voulu d'Auschwitz, qui m'était tombé dessus comme une masse de plomb. Un jour pourtant il faudra que je sache, que j'aie le courage de savoir. (139)

Le discours du maire terminé, quatre jeunes sortent de la maison, intrigués par la petite foule ici rassemblée ; manifestement, ils ne comprennent pas ce qui s'y passe. CA leur dit :

Das war meine Mutter..

Sie wurde deportiert...

Sie wurde ermordet ...

lch habe an ihr keine erinnerung... ${ }^{17}$

«Es ist traurig keine erinnerung zu haben... $»^{18}$, se sent-elle obligée d'ajouter devant leur mine goguenarde :

Ils n'en ont rien à faire, rien du tout, cela ne les regarde pas, ni le passé, ni le présent, ni la mémoire. Ils ont rendez-vous avec des potes au MacDo de la place du marché et tout ce cirque devant leur porte va les mettre en retard.

Je sens le poids de l'inutilité de tout cela, cette remise à jour de la mauvaise conscience d'une génération, nullement partagée par la suivante. Qui baissera les yeux désormais sur ces deux plaques de cuivre? Qui s'en souciera ? (139-140)

\subsection{Le besoin absolu de savoir}

En date du «1er avril 2008 », CA indique que, même si « la tempête » provoquée par les informations qui lui furent fournies à l'automne 2007 par le préposé aux archives de la mairie de Mönchengladbach « s'est apaisée », « le vent continue de souffler sous mon crâne. Riga, Auschwitz ou quoi encore ? Où furent assassinés ma mère et mon frère ? » (105) Et de confier que si

17. «C'était ma mère ... Elle fut déportée ... Elle fut assassinée... Je n'ai pas de souvenir d'elle. »

18. «C'est triste de ne pas pouvoir se souvenir. » 
l'impact d'une telle révélation l'avait incitée tout d'abord à pousser " un cri muet, un désespoir » : « je ne veux pas le savoir !», « maintenant », considérant que sa fuite en avant dure depuis bien trop longtemps, ce qu'elle désire, c'est précisément « savoir » :

Regarder en face ce passé qui continue de tracer des ombres sur ma vie. Connaître la vérité, la transmettre. Qui raconte des bobards et dans quel but ? Pourquoi Hilde Sherman, lors de la rencontre de 1989, m'a-t-elle assuré qu'elle avait très bien connu Henny-Henriette et Gert, qu'ils avaient réussi à survivre jusqu'au bout et que, à l'arrivée des Soviétiques, un SS avait fait un dernier carton en les descendant de quelques coups de feu avant de jeter leurs corps nus dans une fosse improvisée. En 1989, cette version avait peuplé mes nuits de cauchemars. (105)

Une petite vingtaine d'années plus tard, « tout est [donc] remis en question » à la suite du courrier de ce monsieur Winter dont elle n'avait jamais entendu parler :

D'où tient-il ces informations ? En quoi son témoignage peut-il être fiable puisqu'à leurs noms [de sa mère et de son frère] il a ajouté le mien ? Ingeborg Karoline Alexander, prétendil, faisait partie du lot. Comment croire à cette version alors qu'à cette date j'étais en vie. En Belgique. Cachée.

Décidément, comme lors de la rencontre de 1989, je figure à nouveau sur la liste des morts ! Curieuse et récurrente impression de non-être. Peut-être ne suis-je qu'une illusion ? (106)

Certes, le douloureux souvenir que laissa en elle la visite fortuite du camp d'extermination de Dachau à la fin des années cinquante, au retour d'un festival de théâtre universitaire qui s'était tenu à Erlangen, en Allemagne, - « J'accusai le coup sans broncher, sans rien révéler des échos que ce parcours réveillait en moi » (106) - lui avait fait se jurer de ne plus jamais se rendre « dans l'un ou l'autre de ces mémoriaux de barbarie. Pas même celui de Terezin, aux portes de Prague, où fut enfermée ma grand-mère Adèle » : « Je n'ai jamais voulu faire le pèlerinage à Auschwitz, parcourir ce musée de la mort à ciel ouvert, ce cimetière de millions d'âmes envolées en fumée » (106-107). Elle comprend toutefois que les « révélations » de ce monsieur Winter vont l'obliger à éplucher les archives de la Shoah, à questionner les institutions spécialisées d'Allemagne et de Pologne, qu'il lui faudra relire attentivement les 140 pages du livre-témoignage que Sherman lui dédicaça en souvenir de sa mère Henriette : "In Errinerung an 
Henny » : « Je veux savoir. Morgane, ma fille me dit qu'elle veut savoir. La vérité aura le prix de cette douleur remise en éveil. / Briser soixante ans de silence ! ... » (107).

Dans les mois suivants, c'est donc à plusieurs reprises qu'elle consultera Zwischen Tag und Dunkel: Mädchenjahre im Ghetto (Entre le jour et l'ombre : la jeunesse dans le ghetto) (1984), afin de récapituler les événements qui, « un par un, décret par décret », rendirent infernale la vie des Juifs d'Allemagne dans les années trente, de retracer le quotidien des siens et d'elle-même - « Toutes nos errances » (131) -, d'y dénicher des informations qui auraient pu lui échapper lors des lectures précédentes :

chapitre par chapitre, je reconstitue un monde concret où plane l'ombre d'une femme aux jambes magnifiques et celle de son garçon aux yeux sombres. Un monde où mes interrogations continuent de rester sans réponse Je ne comprends pas, je ne comprendrai jamais comment on a pu subir toutes ces humiliations sans réagir, sans fuir, comment des voisins, des amis, des collègues, des copains de classe se transformèrent du jour au lendemain en hyènes ricanantes, en vandales, en voleurs. L'éveil soudain du sadisme le plus primaire chez des gens réputés éduqués, lettrés, pétris d'histoire et de culture.

Puis arriva le jour où les enfants juifs furent carrément interdits d'école, où les hommes, quels que soient leur rang, leur compétence, furent envoyés en usine fabriquer des armes ou expédiés sur des chantiers pour construire les camps d'internement où ils seraient massacrés. Ou les femmes, avec ou sans diplômes, furent parquées dans des hangars de couture pour confectionner des uniformes.

Cette femme seule, aux aguets, attendant en vain un signe de son mari pour pouvoir sauver son garçon si sage, si résigné, qui réclamait la présence de sa petite sœur pour la cajoler.

Page après page, je cherche un signe, un prénom, des initiales pouvant correspondre à cette femme de 39 ans qui était ma mère et à ce garçon de 9 ans qui était mon grand frère. Rien.

Elle y découvre néanmoins que, suite à la rébellion de quelques détenus, le ghetto de Riga fut effectivement fermé en novembre 1943 : l'information " était donc vraie ». Par ailleurs, sur Internet, elle trouve l'état civil complet de son frère parmi les transférés de Riga à Auschwitz, mais nulle trace de sa mère, cette femme dont elle n'a qu'un lointain souvenir : " une silhouette sur le quai d'une gare dans la brume d'un matin de novembre. Le 9 novembre $1939 »(172)$. 
Dans mes nuits blanches et noires, je redeviens souvent foetus au premier étage d'un bordel et je pousse mon premier cri à trois heures du matin. Ma mère m'a guidée sur le quai d'une gare un matin de novembre où il faisait très froid. (178)

Ses voyages à Mönchengladbach l'ont fait, écrit-elle, « remonter aux sources de [s]a vie, à cette absence, ce trou noir que le drôle de libraire à blouse grise avait décelé dans la carte de [s]on ciel » (178). C'est alors qu'elle saisit que la version que Sherman lui raconta vingt ans plus tôt n'était probablement pour cette ancienne rescapée qu' « une forme de consolation » qu'elle désirait lui prodiguer. Aussi, depuis les semaines qui ont précédé la cérémonie de la pose des pierres en souvenir de ces « deux vies massacrées parmi six millions»

- Celle de mon frère qui ne sera pas resté le grand frère protecteur qui m'a toujours manqué. Celle de ma mère qui ne connut jamais le trouble du temps qui passe et vous fait vieillir, ni celui du bonheur de passer comme moi de l'état de mère à celui de grand-mère -,

CA reconnaît être plus que jamais « à la recherche de la vérité sur leur mort » (179).

\subsection{Auschwitz, le lieu de l'indispensable vérité}

À la mi-novembre 2010, au bout de deux années durant lesquelles CA a remisé ses notes « dans la boîte hermétique des questions sans réponse » et cessé, dit-elle, d'y songer, Morgane, bien qu'au courant de la décision de sa mère de ne jamais fouler le sol de Pologne, lui demande de l'accompagner à Auschwitz en guise de cadeau d'anniversaire. Ne pouvant lui refuser « cette chose impensable » et quoiqu'il lui en coûte d'accepter l'idée de ce « pèlerinage »

- Vers quoi ? Pourquoi ce lieu symbole plutôt qu'un autre tout aussi cruel ? Pour moi - et pour Morgane -, tout s'arrête depuis longtemps au mot Riga, capitale de la Lettonie où ma mère fut enfermée avec mon frère. Seule certitude. Tout le reste m'a été raconté dans un maelström de contradictions (182-183) -,

CA doit admettre que, « la boîte des questions sans réponse » s'étant entrouverte, il lui fallut agir et trouver quelqu'un de bon conseil pour organiser un tel voyage (183). Passons sur les détails... Dans l'avion qui, le jeudi 31 mars 2011, les 
emmène de Paris à Cracovie, celle qui continue de s'interroger sur les raisons de ce voyage, apprenant de sa fille qu'elle a été prise d'un malaise vagal - une petite syncope - dès qu'elles ont survolé la Pologne, se dit qu'il lui faut « [être] debout, bien droite pour atterrir sur cette terre haïe » (185). À destination, les deux femmes sont attendues par leur guide Pawel Kozlowski.

Le lendemain, vendredi 1er avril, en route pour Auschwitz, CA laisse vagabonder ses idées : "Qu'est-ce que je suis venue faire ici ? La question ne me quitte pas » (190). Sur place, à la vue des cohortes de touristes qui inondent ce qui fut le théâtre de " la plus vaste organisation industrielle de la mort de l'histoire de l'humanité », elle ne peut que se sentir « de plus en plus étrangère à ce lieu, à ces démonstrations. De quoi au juste ? Mémoire ? Pèlerinage ? Goût du morbide ? » (190). Après la visite de plusieurs blocks : le block 26 réservé aux tatouages - «l'opération obligatoire de fin d'identité humaine pratiquée sur l'avant-bras de chaque nouvel arrivant » (193) -, les blocks 4 à 7 qui regroupent « un ensemble muséal » où " étape par étape » est présentée " la minutieuse reconstitution de l'horreur » (194) - « Des millions d'individus [y] ont fait le parcours de la chair à la poussière, sans distinction d'âge ou d'origine » (195) -, c'est en proie au mal de mer - « le mal de mère peut-être... » (195) - qu'elle s'en retourne vers le block 1 , celui du département des archives, où, quelques heures plus tôt, elle a rempli deux formulaires, un pour Henny-Henriette, un autre pour Gert...

Il est 13 h 50. Je me trouve en un lieu où j'avais juré de ne jamais mettre les pieds. Un lieu symbole qui, je croyais alors, ne me concernait pas directement. Les miens, ma mère, mon frère, c'est ailleurs que je les ai perdus. Je ne sais pas où. Ni quand. Pas à AuschwitzBirkenau où je me trouve poussée par une suite de hasards. (11)

La réponse à la question qu'elle se pose depuis soixante-cinq années tombe soudain de l'imprimante d'un ordinateur. En deux pages que lui tend la préposée. « Où et quand sont morts ma mère Henny-Henriette et mon frère Gert. C'est localisé Daté » (11). Sous 1'intitulé Gedenkbuch - Opfer der Verfolgung der Juden unter der nationalsozialistischen Gewaltherrschaft in Deutschland 1933$1945^{19}$, CA lit, incrédule :

19. Livre commémoratif des victimes de la chasse aux Juifs durant le règne barbare du nationalsocialisme allemand de 1933 à 1945. 


\author{
Alexander, Henriette \\ Geb. Leven \\ 12 Dezember 1902 in Odenkirchen \\ Wohnhaft in München Gladbach \\ Deportation: \\ Ab Düsseldorf \\ 11 Dezember 1941, Riga, Ghetto \\ 02 November 1943, Auschwitz. Vernichtungslager \\ Todesdatum/-Ort: \\ 05 November 1943, Auschwitz. Vernichtungslager ${ }^{20}$ \\ Bundesarchiv I Stand: 10.12.2010
}

Et sur la seconde feuille, les mêmes termes, les mêmes dates, à l'exception du nom :

Alexander, Gert

Geb. 23 Oktober 1932 in München Gladbach. (196-197)

À la lecture et à la relecture des noms, lieux et dates de naissance, CA constate qu'une correction a même été apportée aux données qu'elle-même avait indiquées sur un des formulaires : le lieu de naissance de sa mère n'était pas Mönchengladbach comme elle le croyait, mais Odenkirchen, le village voisin où vivait sa grand-mère Adèle. "Tout le reste est conforme et d'une précision clinique. / Quelque chose se brouille dans mes yeux, dans ma tête. Comment? Pourquoi ? Ici ? Maintenant ! » (197). Elle réalise en effet que cette information sur la mort de sa mère fut enregistrée à Berlin trois mois et demi auparavant ; c'est dire que si elles étaient venues à Auschwitz fin novembre, comme prévu, juste après l'anniversaire de sa fille, et non au printemps - un hasard $?^{21}-$, aucun renseignement n'aurait pu lui être donné, comme ce fut d'ailleurs le cas, sept mois plus tôt, à Pâques 2010, lorsqu'elle formula la même demande « dans les noirs labyrinthes du mémorial de Berlin » (197) où ni les personnes interrogées

20. Alexander, Henriette Née Leven le 12 décembre 1902 à Ondenkirchen. Résidant à München Gladbach. Déportation : de Düsseldorf, le 11 décembre 1941, Riga. Ghetto. 02 novembre: 1943, Auschwitz, camp d'extermination. Lieu et date du décès : 05 novembre 1943, Auschwitz, Camp d'extermination.

21. "'Il n'y a pas de hasard' est un de ses leitmotivs [de Morgane]. Peut-être a-t-elle raison : son souhait en novembre, la date reportée au printemps, l'intervention de Lew [Bogdan] et de Krzysztof [Jasinski, le directeur du Théâtre STU de Cracovie]. Et ce guide imprévu [Pawel] dont on ne voulait pas... Des hasards, vraiment?»(199-200). 
ni les ordinateurs équipés des dernières technologies chercheuses n'avaient pu trouver les réponses aux questions qui obsèdent ses pensées depuis 1946 :

J'avais dix ans quand j'ai su que je ne les reverrais jamais. Alors s'est installée la hantise de les imaginer séparés. Exterminés loin de l'autre, la mère de son fils, alors que moi, la fille, 'la petite', j'étais là à les attendre dans une autre ville, un autre pays. Vivante. Ce vendredi 1er avril 2011, j'ai su enfin qu'ils ne s'étaient pas quittés. (11)

Voici qu'enfín, dans ce lieu où j'avais juré de ne jamais mettre un pied, vient de tomber la réponse à mon interminable quête, à toutes les suppositions faites sur le sort de l'un ou de l'autre, ensemble ou séparés, à toutes les parcelles d'informations contradictoires recueillies pendant 65 ans ! (197)

«Une lumière m'inonde », exulte CA qui, pour la première fois depuis ces jours de l'après-guerre en Belgique, peut enfin retracer le trajet qu'ont suivi côte à côte sa mère et son frère - « Ils sont morts ensemble. Ils ne se sont pas quittés, du premier au dernier jour »-depuis leur arrestation à Mönchengladbach jusqu'à leur arrivée à Birkenau où ils empruntèrent tout aussitôt « le chemin de la mort » tel qu'elle-même vient de le découvrir dans la maquette en stuc gris du Block 4 : « Je vais pouvoir graver une date sur la plaque de marbre noir du petit cimetière juif d'Odenkirchen-Rheydt» (198).

\begin{abstract}
Morgane me prend la main, la serre. Nous sourions et pleurons tout à la fois. Des larmes discrètes, sans honte, sans retenue. Nous voici libérées ! Un poids immense s'envole dans les étages de cet ancien bordel ${ }^{22}$ ! Un lieu décidément prédestiné, puisque je suis née, parait-il, au premier étage d'un autre bordel. (198)
\end{abstract}

Pour sa fille aussi qui, depuis sa prime jeunesse, connaissait son histoire familiale et qui trop tôt sut le prix que d'aucuns, comme sa mère, eurent à payer pour le simple fait d'être juifs, « le monde a changé »: dans « ces lieux de l'indicible » qu'elles ont découverts bras dessus bras dessous, « brusquement, ce séjour a pris tout son sens »(199). Le lendemain 2 avril, lors de la visite de Cracovie, après une nuit où elles purent dormir «paisiblement » - «pour la première fois depuis des semaines et des mois de peurs et d'angoisses, chez

22. Pendant la guerre, au premier étage du block 1 - l'actuel bâtiment des archives - se trouvait en effet un bordel réservé aux officiers allemands mais où de simples soldats pouvaient entrer s'ils avaient été zélés ; « la porte des plaisirs » était également ouverte, de temps en temps, « aux kapos les plus serviles » (191). 
l'une comme chez l'autre »-, elles prennent enfin « le temps d'avoir du temps à deux, à [elles] seules, mère et fille, liées par une complicité d'un nouveau type » (205-206).

La visite, le dimanche matin du 3 avril, de Birkenau - l'agrandissement et la zone finale d'Auschwitz - et des alentours du camp où, apprennentelles, mille Juifs en provenance de Riga débarquèrent le 5 novembre 1943 850 furent immédiatement acheminés vers les chambres à gaz -, leur permet désormais d'imaginer Henny et Gert entrant « dans cet endroit maudit dont il ne reste que cette pierraille morcelée » (214), de voir l'emplacement des fours crématoires où ils partirent " en fumée » (201) ainsi que les stèles de marbre noir, rangées trois par trois sur les emplacements des fosses communes ou d'incinération en plein air. Une phrase identique y est gravée en plusieurs langues : À la mémoire des hommes, des femmes et des enfants tombés victimes de la barbarie (213). Face aux crématoires en ruine, CA ne peut se retenir de laisser affluer en elle de déchirantes questions sur le moment de leur arrivée et des instants ultérieurs :

Avaient-ils des bagages ? Que transportaient-ils que les SS allaient confisquer, trier, redistribuer ? Comment étaient-ils habillés ? Comment était coiffée ma mère après deux années passées à Riga, elle qui fut si belle et si coquette ? J'imagine leurs minces silhouettes se faire dépouiller, dénuder, raser avant d'être poussées vers la pseudo salle de douche où une rangée de nazis zélés les attendent, faisant la haie le long d'une paroi. Ils les surveillent en se glissant dos au mur au fur et à mesure du 'remplissage' de la salle, et quand il n'en reste plus aucun dehors, ils se dépêchent d'en verrouiller la porte et sortent au pas de course. Les fins galets de Zyklon B sont jetés par des fentes du plafond, ils dégringolent jusqu'au sol, se dissolvent au contact de la peau. L'étouffement part du bas. C'est la fin du calvaire, la fin de la fin.

Pas pour tous. Le corps de Henny, ma mère, fut-il soumis à une deuxième séance de rasage du pubis et des poils sous les bras ? Avait-elle une dent en or qu'on lui aurait arrachée comme on arrache une tique dans le poil d'un chat ? Où furent déversées ses cendres et celles de son enfant? Dans le pré avoisinant ou dans la petite mare où coassent des grenouilles ? (214-215)

L'après-midi, la visite du Mémorial leur procurera un moment de grande émotion, lorsque le public entonnera la Hatikva, l'hymne national israélien. Plus tard, par un heureux hasard - "Hasard encore ? ou autre chose ?»-, elles tomberont sur Ida Grinspan et l'équipe de tournage venue filmer le témoignage 
de celle qui à 14 ans fut déportée à Birkenau, elle que Morgane avait rencontrée à plusieurs reprises et avec qui elle avait sympathisé : d'ailleurs, n'est-ce pas Ida « qui l'avait encouragée à sauter le pas de cette visite taboue » (220) ?

Après ce «plein de pèlerinage "- « Nous savons, Morgane et moi, que l'envie nous reprendra un jour de revenir dans ce pays où nous avions juré de ne jamais mettre les pieds » (227) -, c'est dans une joyeuse ambiance que s'effectue, le 5 avril, le retour à Paris où, malgré la reprise de la vie quotidienne, «plus rien n'est comme avant. Quelque chose dans ma vie a définitivement changé » (229), confie CA :

Une pelote de questions désormais s'est dénouée, depuis ce vendredi 1er avril, à $13 \mathrm{~h}$ 50, dans le bureau des archives d'Auschwitz. Henny, Henriette, ma mère, n'est plus une abstraction, une inconnue disparue dans un point d'interrogation. Toutes les hypothèses échafaudées durant 65 ans, parfois en espoirs déraisonnables, en rêves fous, se sont enfin dissipées. [...]. Ainsi, ce 1er avril 2011, ma hantise d'imaginer séparés ma mère et mon frère est tombée d'un seul coup d'œil à la lecture de deux feuilles sorties d'une imprimante. Tout est devenu réel. Je peux reconstituer, pour ainsi dire pas à pas, cette journée particulière du 5 novembre 1943, depuis le débarquement sur la Judenrampe jusqu'à l'entrée du crématoire, III ou IV, qu'importe. Ils ont pris vie en moi pour la première fois depuis 65 ans. J'ai marché sur les chemins empruntés, je sais le déroulement étape par étape, salle par salle, jusqu'aux cendres jetées dans une mare. Ma mère est enfin une femme de chair et non une simple idée de mère. Je la sens physiquement, comme je sens la présence de l'enfant qui était mon frère aîné et qui venait d'avoir 11 ans et 10 jours quand il a été exterminé. Ma mère aurait dû atteindre la belle quarantaine, un mois et une semaine plus tard. Le 12 décembre. Signe du Sagittaire Signe de feu. (229-230)

\section{3. Épilogue}

Désormais, quand elle passe par le boulevard Saint-Martin où se trouvait autrefois le théâtre de l'Ambigu, CA ne peut s'empêcher de penser au libraire trappiste-fasciste, celui qui, un demi-siècle plus tôt, se lamentait que les Allemands n’aient pas gagné la guerre,

leur juste guerre !, disait-il. Elle serait enfin libérée la petite Belgique, et la France et le reste du monde, libérée des juifs et de toutes les autres nuisances. Et il y aurait enfin de l'ordre, car ces gens-là, mademoiselle, les Allemands, avaient le sens de l'ordre, je peux vous l'assurer ! 
[...] L'Occident a perdu sa seule chance de salut, mademoiselle. S'ils étaient restés, les Allemands, ils nous auraient débarrassés de toutes ces racailles. (77)

Aujourd'hui, combien elle aimerait pouvoir lui dire avec un grand sourire : "Vous savez, ce trou noir dans mon ciel, ce vide astrologique qui vous intriguait tant, moi, je l'ai identifié tout seule. Sans l'aide de vos astres »(231)! Un témoignage qui rejoint en quelque sorte celui de l'écrivaine et psychanalyste Lydia Flem dont la famille fut elle aussi durement frappée par la tragédie de la Shoah :

\footnotetext{
Toute une vie pour essayer de la résoudre. Pour devenir l'auteur, non pas de ses jours, mais de son propre récit. Raconter l'histoire à sa manière. Prendre du recul; entre actions et réactions, dessiner son propre chemin. Tenter d'être soi. Toute une vie pour devenir qui on est. (2009: 34)
}

Comme l'écrit très justement Pierre Mertens, assurément «si les nazis pouvaient lire aujourd'hui des livres de cet ordre, ils mesureraient l'ampleur de leur vraie défaite : car ils n'ont pas réussi à tuer chez certains ce goût d'un bonheur invincible » (8). De fait, ajoute-t-il,

la voix sourde, mais assurée de Caroline Alexander ne laisse jamais entendre le moindre accent de ressentiment, mais elle excelle à nous rendre sensible la joie d'avoir survécu et le bonheur d'éprouver des sentiments humains, au sein d'un monde qui réussit, un temps, à le faire oublier. (9)

\section{Références bibliographiques}

Alexander, C. (2014). Ciel avec trou noir. Bruxelles : Éditions MEO (avec une préface de P. Mertens: 5-9).

Alexander, C. (2018). Une vie en miniature. Roman (d'amour et de chats). Bruxelles : Éditions MEO.

Audétat, M. (2015). « Je constate que la Shoah suscite désormais une forme de lassitude » (Nathalie Skowronek). Le Matin Dimanche, 12 avril 2015: 17.

Binet, L. (2009). HHhH. Paris : Grasset.

Cyrulnik, B. (1999). Un merveilleux malheur. Paris : Odile Jacob.

Cyrulnik, B. (2019). La nuit, j'écrirai des soleils. Paris : Odile Jacob, coll. OJPsychologie. 
Flem, L. (2009). Comment je me suis séparée de ma fille et de mon quasi-fils. Paris : Seuil, coll. Points.

Lafon, Fr. (s.d.). "Wagner en guise de vaccin », Le Cabinet des curiosités " $L a$ musique est partout, à nous d'aller la chercher", Muzikzen. <https://www. meo-edition.eu/ciel.html> (Accès 16 décembre 2019).

Rouhart, M. (s.d.). « Caroline Alexander, Une vie en miniature, roman, MEO », $<\mathrm{https}$ ://www.areaw.be/caroline-alexander-une-vie-en-miniature-romanmeo $>$ (Accès 16 décembre 2019).

Skowronek, N. (2015). " Auschwitz. Les gardiens de la mémoire. Un entretien avec Nathalie Skowronek », Information juive <https://docs.wixstatic. com/ugd/7d530a_13599aa812d74a87b49b679e55c23215.pdf> (Accès 16 décembre 2019).

Skowronek, N. (2015). La Shoah de Monsieur Durand. Paris : Gallimard.

Todorov, T. (2004). Les abus de la mémoire. Paris : Arléa.

Wieviorka, A. (2011). L'heure d'exactitude: Histoire, mémoire, témoignage. Entretiens avec Séverine Nikel. Paris : Albin Michel, coll. Itinéraires du savoir. 